\title{
Renal Transplant Pathology Series-III (A)
}

\section{Dr. Muhammed Mubarak}

Professor of Pathology and HOD

JIK Department of Histopathology,

Sindh Institute of Urology and Transplantation (SIUT),

Karachi, Pakistan.

Email address: drmubaraksiut@yahoo.com

PJKD 2020;4(3):297-303

Banff classification of renal allograft pathology has evolved substantially over the past 30 years and has truly become a multidisciplinary activity. It is no longer a one man' s (especially histopathologist' s) job to accurately diagnose and classify renal transplant pathology, especially rejection. With this, it has also increased in complexity and level of difficulty. In this pictorial, we aim to provide an illustrated presentation of the Banff classification categories and practical tips and tricks to identify and report the lesions. This will be helpful for better understanding of the renal transplant pathology not only for trainees and residents of nephrology and histopathology but also practicing pathologists and nephrologists.

\section{Banff category 2. Antibody-mediated changes}

This category has undergone the most profound changes during the evolution of Banff classification, reflecting the growing body of knowledge and clinical experience in this field. Even the nomenclature of the category has been changed several times, as shown in Table 1.

Table 1. Main changes in the nomenclature and classification of antibody-mediated rejection.

\begin{tabular}{|c|c|}
\hline Meeting Reports, years & Category 2. Antibody mediated rejection \\
\hline Pre-Banff & Hyperacute rejection \\
\hline Banff ‘93 & Hyperacute rejection \\
\hline Banff ‘97 & $\begin{array}{l}\text { Antibody-mediated rejection } \\
\text { 1. Hyperacute } \\
\text { 2. Accelerated acute }\end{array}$ \\
\hline Banff '97 update & $\begin{array}{l}\text { Antibody-mediated rejection } \\
\text { Type I. ATN-like } \\
\text { II. Capillary } \\
\text { III. Arterial }\end{array}$ \\
\hline Banff '07 & $\begin{array}{l}\text { Antibody-mediated changes (may coincide with categories } 3,4 \text { and } 5 \text { and } 6 \text { ) } \\
\text { C4d deposition without rejection } \\
\text { Acute antibody-mediated rejection } \\
\text { Chronic active antibody-mediated rejection }\end{array}$ \\
\hline Banff '13 & $\begin{array}{l}\text { Antibody-mediated rejection } \\
\text { Acute/active ABMR } \\
\text { Chronic active } \\
\text { C4d-negative ABMR }\end{array}$ \\
\hline Banff'15 & $\begin{array}{l}\text { Antibody-mediated changes } \\
\text { Acute/active ABMR } \\
\text { Chronic active ABMR } \\
\text { C4d staining without evidence of rejection } \\
\text { Transplant arteriopathy may be seen in chronic ABMR }\end{array}$ \\
\hline Banff'17 & $\begin{array}{l}\text { Antibody-mediated changes } \\
\text { Active ABMR } \\
\text { Chronic active ABMR } \\
\text { C4d staining without evidence of rejection } \\
3 \text { criteria for ABMR diagnosis remain but C4d can substitute for DSA. } \\
\text { DSA testing still advised }\end{array}$ \\
\hline Banff'19 & $\begin{array}{l}\text { Category 2: Antibody-mediated changes } \\
\text { Active ABMR } \\
\text { Chronic active ABMR } \\
\text { Chronic (inactive) ABMR } \\
\text { C4d staining without evidence of rejection }\end{array}$ \\
\hline
\end{tabular}

The changing nomenclature reflects the changing knowledge and understanding of the role of antibodies in causing injury to renal allografts. For example, in the Banff 93 and 97 classification, the category was named as hyperacute rejection (HAR). This was because, at that time, it was widely thought that antibodies cause only this type of rejection. As a corollary to this widespread viewpoint, the focus 
of first few Banff meetings was on the diagnosis and classification of cellular rejection and antibodies received little attention of the researchers and clinicians.

Beginning from Banff 2001 meeting, however, the focus of Banff classification shifted to antibody-mediated rejection (ABMR) and the name of this category was changed to ABMR. It was acknowledged that antibodies can also cause acute rejection instead of just HAR or accelerated acute rejection. This was the result of increasing knowledge of and the burgeoning literature regarding the role of antibodies in the rejection process. Note that from Banff 2015 onwards the name of the category was changed to antibody-mediated changes, rather than ABMR, because it has been hypothesized that antibodies may be involved in accommodation and the full spectrum of antibody actions and effects on graft parenchyma remain unexplored. The current nomenclature reflects the broad spectrum of changes that can be mediated by antibodies in the graft, many of which remain to be explored.

A number of factors were behind increased focus on ABMR: (i) increased recognition of the condition, (ii) better techniques for detecting antidonor antibodies, (iii) increasing numbers of re-transplants, i.e., second or third transplants and (iv) increased transplantation across $\mathrm{ABO}$ and other immunologic barriers as a result of shortage of donor organs. The identification of some relatively specific pathological features and one component of complement for antibody-mediated rejection, i.e., C4d, also helped revive interest in and increased recognition of $A B M R$.

It is not possible to cover the entire topic of antibody-mediated changes in just one installment. It will be presented in three parts. This is the first part of it and discusses HAR and accelerated acute rejections. Fortunately, both of these types of rejections are rarely seen nowadays. However, their diagnosis is relatively straight forward and can be made just on morphological features alone, which are very characteristic and are shown in series of figures below. In addition, clinical picture is also distinctive. As you will see, the main target of ABMR is the vascular compartment, mostly microcirculation (glomeruli and peritubular capillaries), but larger arteries may also be involved. This is understandable as the antibodies circulate in the blood stream and they first come in contact with the endothelium of the blood vessels and target it for injury. The nature of the pathological changes are variable and depend on the time period elapsed before the biopsy is examined, as you will see in the following images.

A series of representative images of biopsies with category 2 of antibody-mediated rejection are illustrated in Figures 1 to 6.

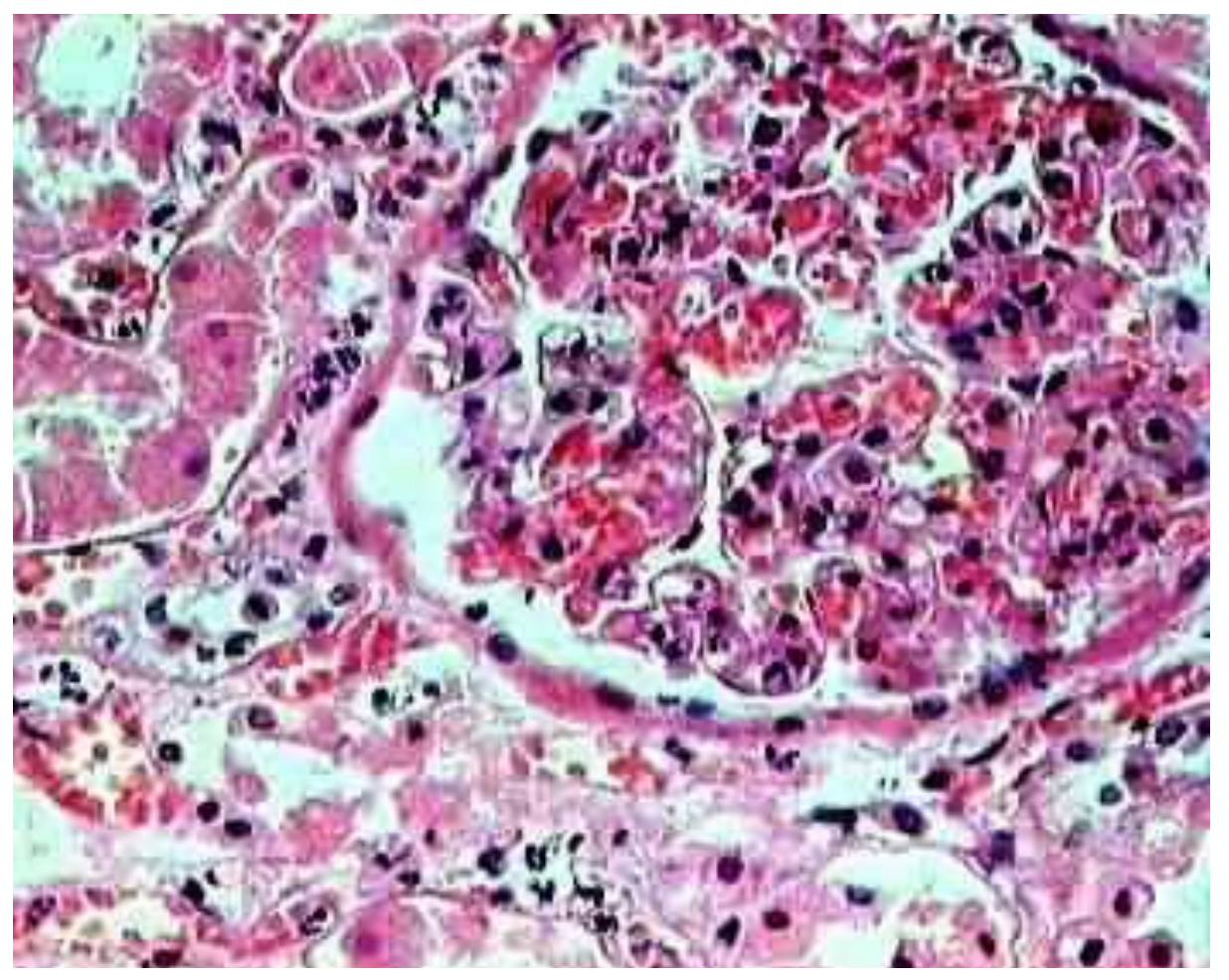

Figure 1. High-power photomicrograph showing a representative area of a renal allograft which was removed due to the development of hyperacute rejection.

The glomerulus shows stagnation and clumping of red blood cells in many capillaries. In addition, many capillary Lumina contain polymorph neutrophils. A few peritubular capillaries are also visible and show dilatation and neutrophils in the lumina, a lesion known as pertitubular capillaritis. Severe acute tubular injury (ATI) is also seen. This represents relatively early phase in the development of hyperacute rejection (H\&E, $\times 400)$. 


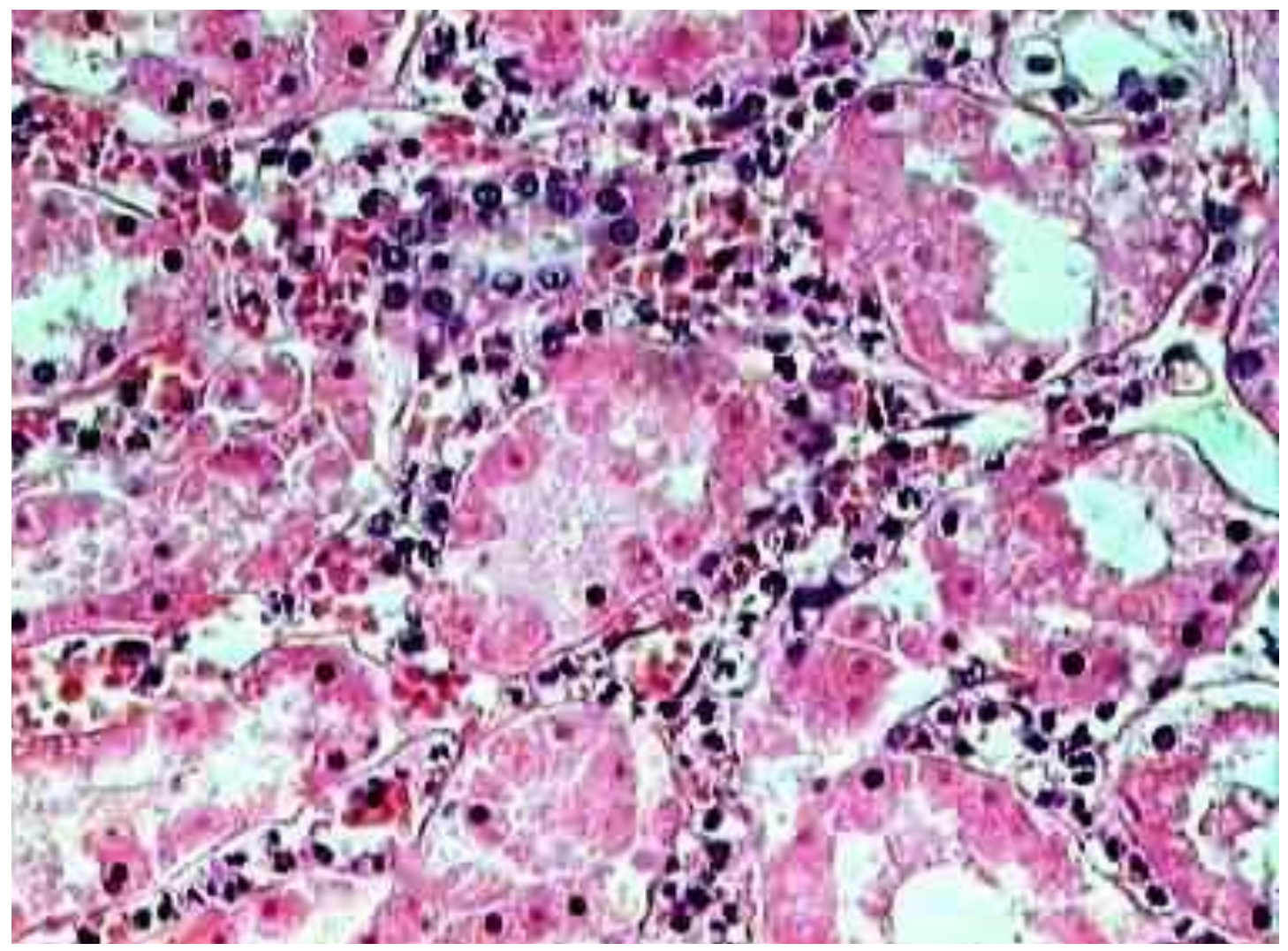

Figure 2. High-power photomicrograph showing a representative area of a renal allograft parenchyma comprising of tubule-interstitial compartment from a graft which was removed due to the development of hyperacute rejection.

The peritubular capillaries are prominent and show dilatation and neutrophils in the lumina, a lesion known as pertitubular capillaritis. Severe acute tubular injury (ATI) is also seen.

This represents tubule-interstitial counterpart of the changes occurring in the glomeruli as shown in Figure 1 and occur during relatively early phase in the development of hyperacute rejection (H\&E, $\times 400)$. 


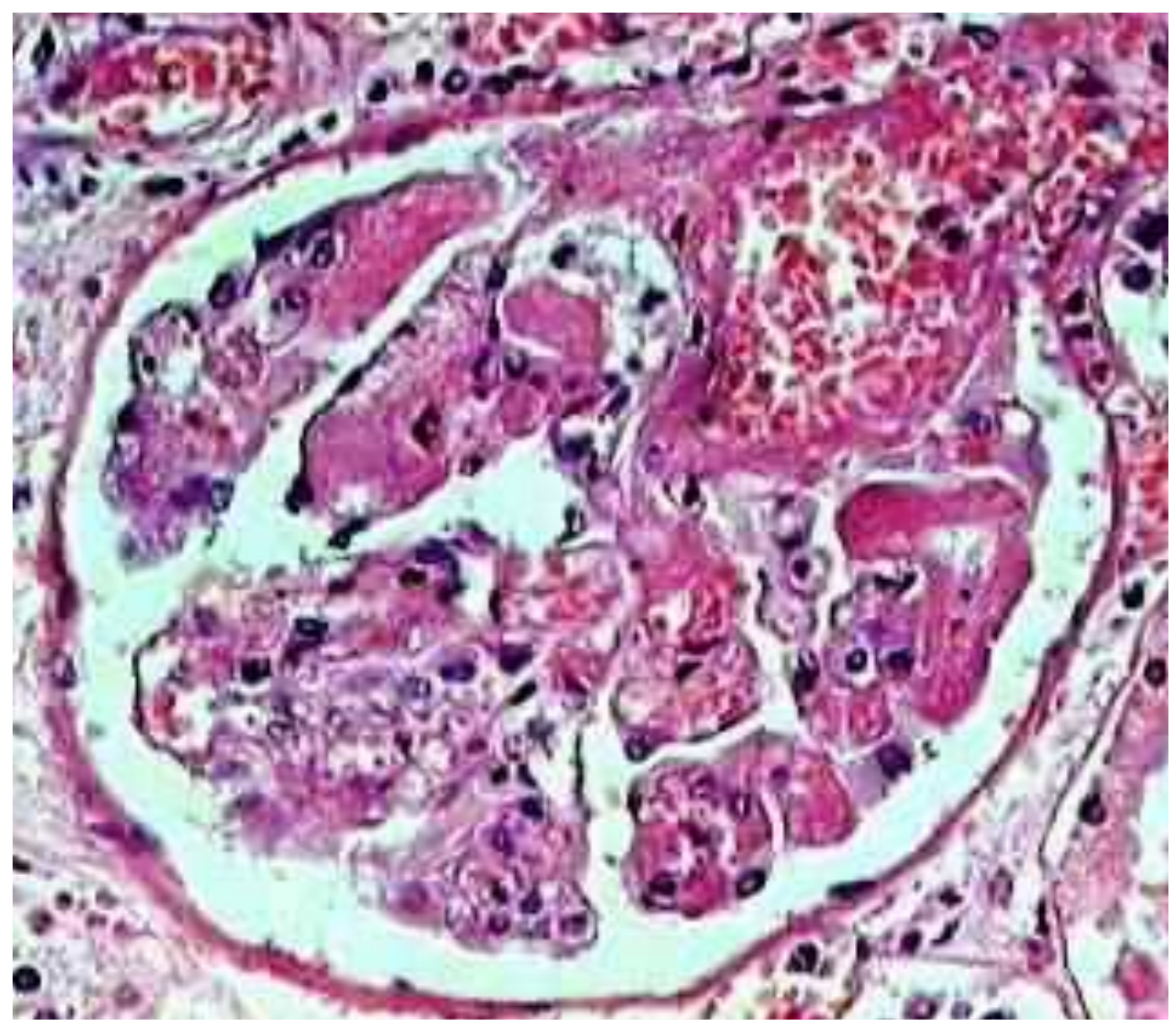

Figure 3. High-power photomicrograph showing a glomerulus from a renal allograft which was removed due to the development of hyperacute rejection.

The glomerulus still shows stagnation and clumping of red blood cells mainly in dilated arterioles at hilar pole. In addition, many capillary lumina contain fibrin thrombi, a lesion called thrombotic microangiopathy and some capillary lumina also contain a few polymorph neutrophils.

This represents relatively delayed phase in the development of hyperacute rejection (H\&E, $\times 400)$. 


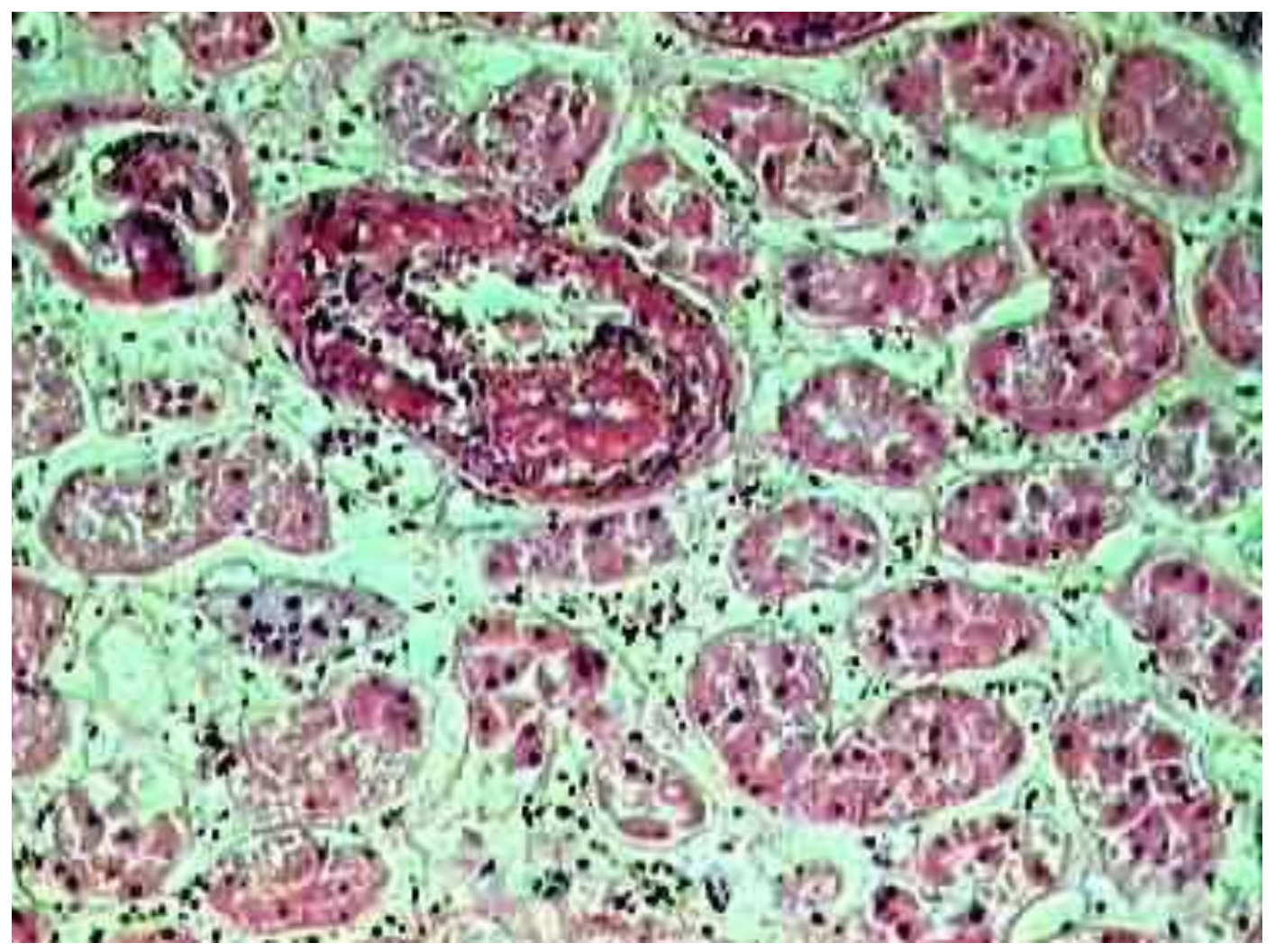

Figure 4. This is medium-power photomicrograph of a representative area from a renal allograft which was removed due to the development of hyperacute rejection.

There is one small artery in the center of the field showing fibrinoid necrosis and transmural inflammation ( $\mathrm{v} 3$ lesion). This is highly suggestive of antibody-mediated rejection but can also be seen as part of T cellmediated rejection.

In addition, there is generalized interstitial edema and foci of peritubular capillaritis. Tubules show severe acute tubular injury (ATI) but nuclei are still present.

This also represents relatively delayed phase in the development of hyperacute rejection $(H \& E, \times 200)$. 


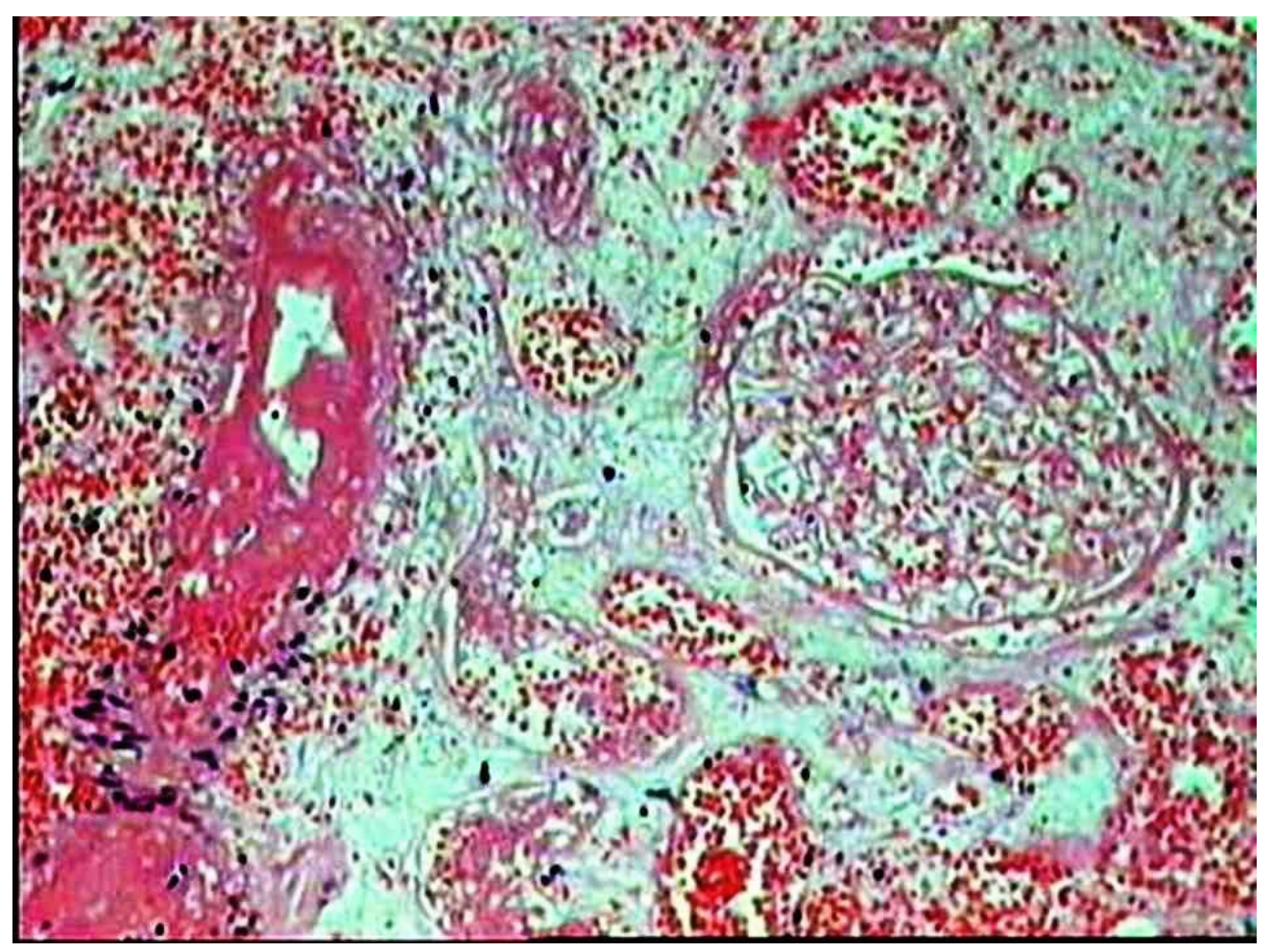

Figure 5. This is medium-power photomicrograph of a representative area from a renal allograft which was removed due to the development of hyperacute rejection.

There is one small artery showing fibrinoid necrosis of the wall (v3 lesion). In addition, there is almost complete infarction of the graft parenchyma (including tubules and glomerulus) and severe congestion.

There is also generalized interstitial edema. This represents fairly advanced phase in the development of hyperacute rejection. Obviously, such a graft has to be removed. $(H \& E, \times 200)$. 


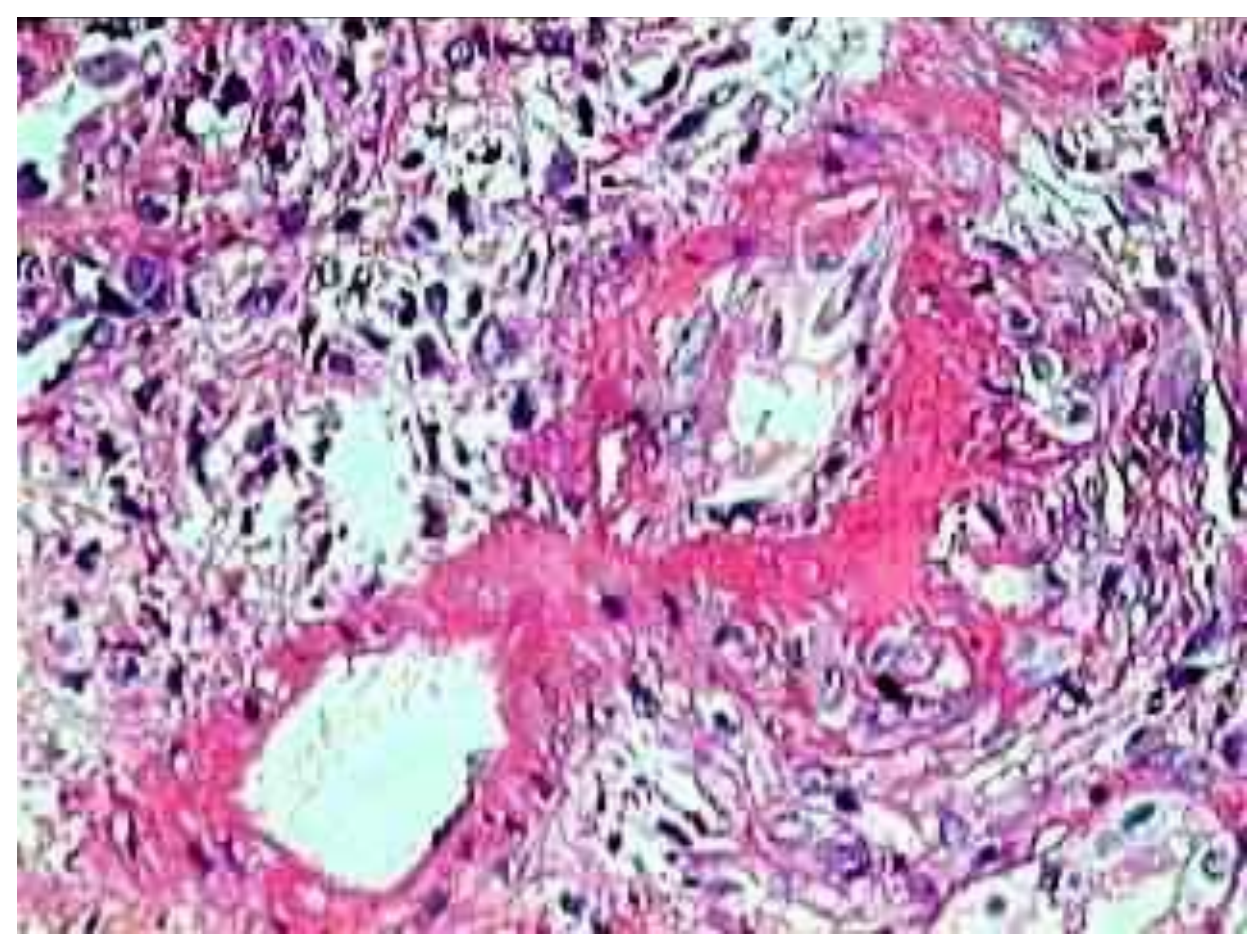

Figure 6. This is medium-power photomicrograph of the biopsy with an interlobular artery at the center of the field. This amount of fibrous tissue is normal surrounding larger vessels.

There is extensive fibrinoid necrosis of the arterial wall. This lesion qualifies for $\mathrm{v} 3$ Banff lesion score and is typically associated with antibody-mediated rejection (H\&E, $\times 200)$.

\section{Further reading:}

1. Mubarak M, Kazi JI. Evolution of the diagnostic criteria of antibody-mediated rejection of renal allografts: Banff classification updates. Port J Nephrol Hypert 2013; 27: 137-42.

2. Racusen LC,Colvin RB,Solez K, Mihatsch MJ, Halloran PF, Campbell PM, et al. Antibody-mediated rejection criteria - an addition to the Banff 97 classification of renal allograft rejection.Am J Transplant 2003;3(6):708-714.

3. Loupy A,Haas M,Solez K,Racusen L,Glotz D,Seron D, et al. TtheBanff2015kidneymeetingreport: current challenges in rejection classification and prospects for adopting molecular pathology. Am J Transplant 2017;17:28-41.

4. Roufosse C, Simmonds N, Clahsen-van Groningen M, Haas M, Henriksen KJ, Horsfield C, et al. A 2018 reference guide to the Banff classification of renal allograft pathology. Transplantation 2018;102:1795-1814. 